\title{
Waveform-Based State Determination for Catalytic Converters
}

\author{
lurii Motroniuk, Ralf Stöber, Gerhard Fischerauer \\ Chair of Metrology and Control Engineering, Universität Bayreuth, 95440 Bayreuth, Germany \\ mrt@uni-bayreuth.de
}

\begin{abstract}
:
A novel microwave-based approach for monitoring the state of aftertreatment systems such as diesel particulate filters (DPF), three-way catalytic converters (TWC), and selective catalytic reduction (SCR) catalysts is proposed. The volume inside the metallic housing of the DPF, TWC, or SCR is considered as a wireless communications channel between two terminals of a communications system. It is shown that, depending on the transmission channel characteristics, the properties of the catalyst, such as the catalyst state, can be inferred. This is done by means of an ultra wideband (UWB) measurement and the subsequent evaluation and processing of the waveform.
\end{abstract}

Key words: microwave-based, catalyst state, ultra wideband, communications channel, waveform.

\section{Introduction}

Modern regulations for exhaust gas components like Euro 5 and Euro 6 require new methods both for the emissions control and for the monitoring and operation of the emission control systems in the diesel and gasoline engines.

During diesel engine operation, soot is captured in the pores of the DPFs or the SCR catalysts. The ceramic filters must be periodically regenerated to avoid blocking. Hence, a knowledge about the soot load is useful for the engine control in a number of ways $[1,2,3]$.

For gasoline engines, a TWC is used to keep the polluting emissions low. The TWC stores and releases oxygen depending on the operating conditions. As in the DPF case, it would be of great advantage from the control engineering point of view to know the inner state of the catalyst and especially the oxygen loading state. However, it is the state of the art that the loading state cannot be measured directly, but is derived indirectly from the oxygen concentration in the exhaust gas stream before and after the catalytic converter. The oxygen-measuring elements (so-called lambda probes) are implemented for the loading state estimation [4].

In a recent conference contribution, we have described a novel approach towards the monitoring of exhaust gas aftertreatment systems such as DPFs, SCRs, and TWCs [5]. It was proposed to consider the interior of the catalyst metal housing as a wireless communications channel. The effect of the catalyst state on such communication parameters as the bit error rate (BER), the packet error rate (PER), the ratio of the energy per bit to the noise power spectral density $E_{b} / N_{0}$ and the data receive rate ( $\mathrm{Rx}$ data rate) was demonstrated by way of an example. The described approach involves a small-size and cost-effective modular architecture and has the potential for field application (i.e., inside a vehicle) owing to space and cost reasons, unlike approaches based on laboratory equipment, such as automatic vector network analyzers [6].

In this paper, we propose a modification of our novel approach. Instead of measuring certain communication channel parameters (such as BER, PER, $E_{b} / N_{0}$ levels, Rx data rate), an UWB pulse is to be transmitted through the catalyst, and the changes suffered by it on its way through the catalyst are to be evaluated by a receiving device. In other words, the propagation medium characteristics and the catalyst state are to be estimated from the UWB waveform changes.

The rest of the paper is organized as follows. In Section II, a novel wireless approach is presented, followed by a description of a hardware implementation and of an experimental verification with DPF in Sections III and IV, respectively. The conclusions are presented in Section V. 


\section{Novel Wireless Approach}

It is proposed to consider the interior of the catalyst housing as a communications channel between two terminals of an ultra wideband (UWB) wireless communications system. The most important parameters during the data transmission are the characteristics of the communications channel (in our case the catalyst). From the wireless channel characteristics, the properties of the catalytic converter such as the catalyst oxygen concentration or soot loading state can be determined.

The basic structure of the proposed measurement system is shown in Fig. 1. The system consists of a conductive (usually metallic) housing of the catalyst (1) and the catalyst itself (2). Two gas-permeable conductive grids $(3,4)$ which limit the resonating cavity are of advantage, but not absolutely necessary. The grids are effectively short-circuiting the electric field, thus precisely defining the resonating cavity. Two thin-probe feeds (short stubs 5,6 ) serve to connect the cavity with the system environment, i. e., with communication end devices $(7,8)$, by coaxial cables, which are used for signal transmission and reception. The short stubs play the same role for the communications system as the antennas in the common message transmission and the catalyst in its housing plays the same role as the wireless communication channel for the ordinary message transmission.

The signal transmitted through the wireless channel inside the catalyst suffers from such effects as attenuation, fading, multipath propagation, scattering, etc. Note, however, that the sender and receiver in a common wireless transmission link are located in the far field of each other whereas, in the present context, they are located in the near field. This, together with the resonance phenomena in the catalyst-filled cavity, introduces new propagation-path effects compared to wireless transmission links. The characteristics of the received information transferred from the transmitter to the receiver depend on the propagation medium. The effect on the signal is similar to the effect of buildings, trees, autos,

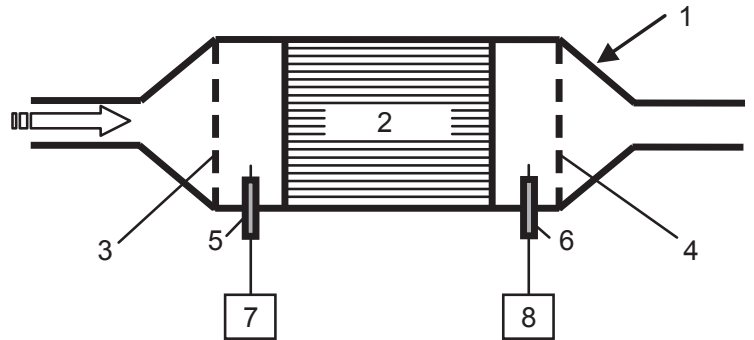

Fig. 1. Basic structure of the proposed measurement system.

rain, humidity, etc. in common wireless communication links: the channel characteristics influence the signal waveform received. Here, the closed-space propagation through the soot-loaded or otherwise chemically altered catalyst medium affects the received signal waveform.

In this paper, we specifically focus on a UWB communications system. Rather than measuring individual channel parameters, such as BER, PER, $E_{b} / N_{0}$ levels, or the Rx data rate as in [5], or than measuring the S-parameters of a microwave two-port as in [6], it is the integral UWB pulse waveform transmitted through the device under test (catalyst) which is considered. It is postulated that physical changes of the catalyst material caused by the absorption or desorption of soot or chemical species are mirrored in the waveform received.

The goal, of course, is to estimate the internal state of a catalyst by this approach in a manner substantially simpler than possible with the system architectures demonstrated so far. This is achieved because the measurement can be performed by the existing hardware (wireless communication modules) and software (signal processing techniques for the wireless communication systems).

The functional diagram of such a system is shown in Fig. 2. A pulse generator generates the UWB waveform, which is then transmitted through the device under test (catalyst) and distorted in this process. On the receiver side, the UWB pulse waveform is measured to indirectly estimate the catalyst state.

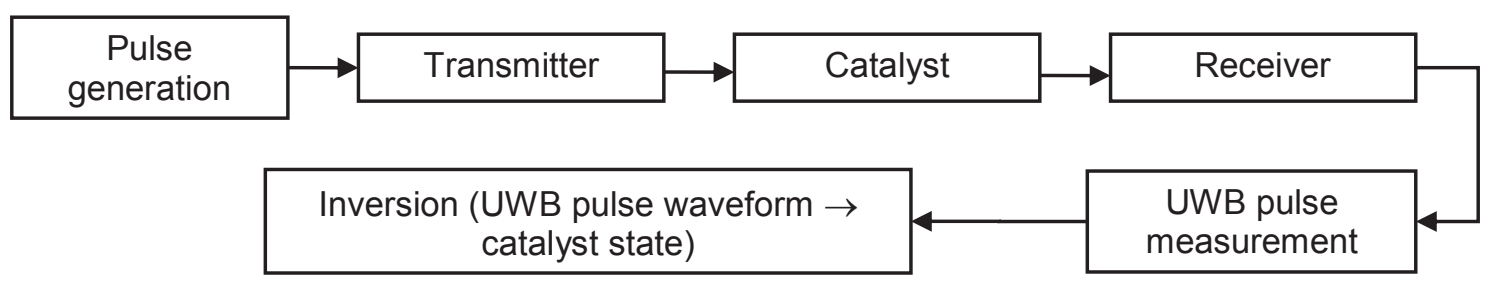

Fig. 2. Functional diagram of the proposed measurement system. 


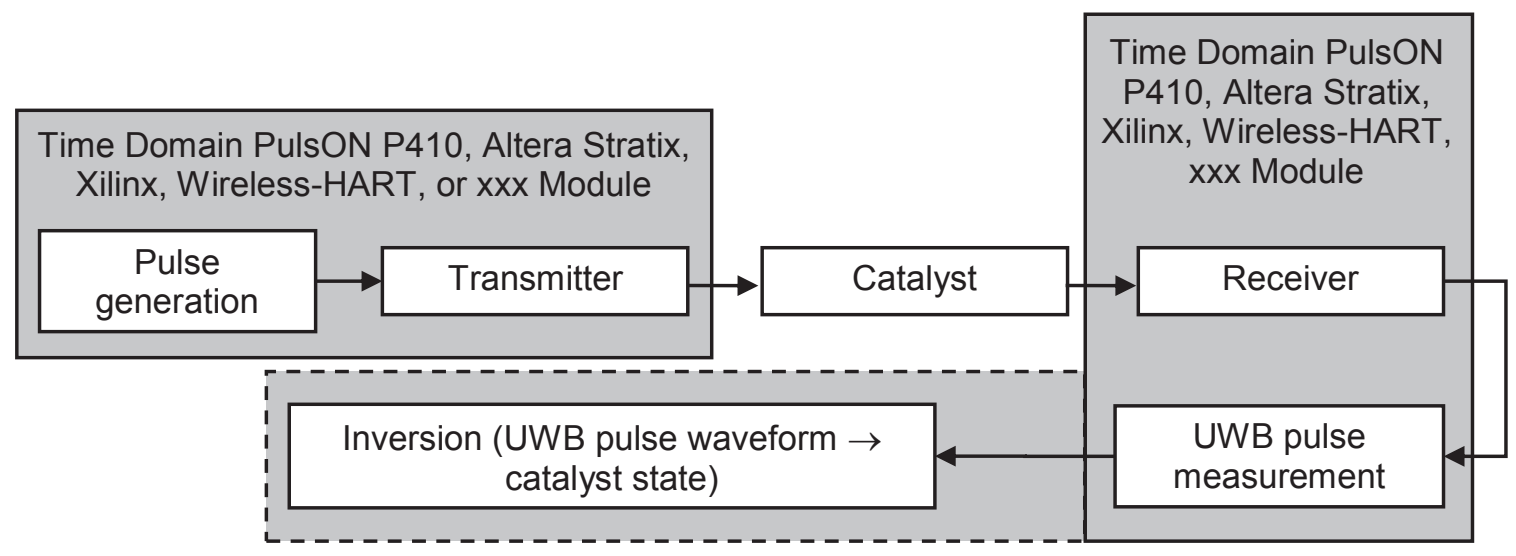

Fig. 3. Exemplary hardware implementation of the proposed measurement system.

\section{Hardware Implementation}

Fig. 3 shows some possible ways to implement the architecture proposed in actual hardware. The UWB pulse generation and the transmission are accomplished by a communications module (we have used a Time Domain PulsON P410 module), and the receiver is implemented by another communications module (PulsON P410, too). After receiving the pulse modulated by the catalyst properties, it is a matter of experience (calibration, simulation results, ...) to infer the catalyst state from the waveform distortion.

Devices such as Altera Stratix, Xilinx Virtex, Xilinx Spartan, ZigBee, Wireless HART, Nanonet, PulsON 410, or any other including (but not limited to) field-programmable gate array (FPGA) based integrated circuits and devices could be used for building an appropriate communications system for the intended application purpose. The catalyst state can be estimated by a post-processing of the received UWB waveform with the numerical computing environments and programming languages (such as MATLAB, C, etc.).

\section{Experimental Verification with DPF}

The validity of the approach was tested with DPFs loaded with different amounts of soot. The aim of the tests was to compare the effect of the soot load on the communication channel and, as a result, on the received UWB waveforms in order to prove the validity of the, as we believe, novel approach towards the monitoring and estimation of catalyst states.

One of the DPFs was clean (not used, with $0 \mathrm{~g}$ of soot), another one was loaded with $4.6 \mathrm{~g}$ of soot, and the third one was loaded with $13.6 \mathrm{~g}$ of soot. The filters used and their soot loading are listed in Tab. 1. For details of the catalyst geometry, the reader is referred to [7].
Tab. 1. Soot load of the measured DPFs [7].

\begin{tabular}{lcc}
\hline $\begin{array}{l}\text { DPF } \\
\text { number }\end{array}$ & $\begin{array}{c}\text { Soot load } \\
\text { in g }\end{array}$ & $\begin{array}{c}\text { Soot load per DPF } \\
\text { volume in g/L }\end{array}$ \\
\hline 1 & 0 & 0 \\
2 & 4.6 & 2.0 \\
3 & 13.6 & 5.8 \\
\hline
\end{tabular}

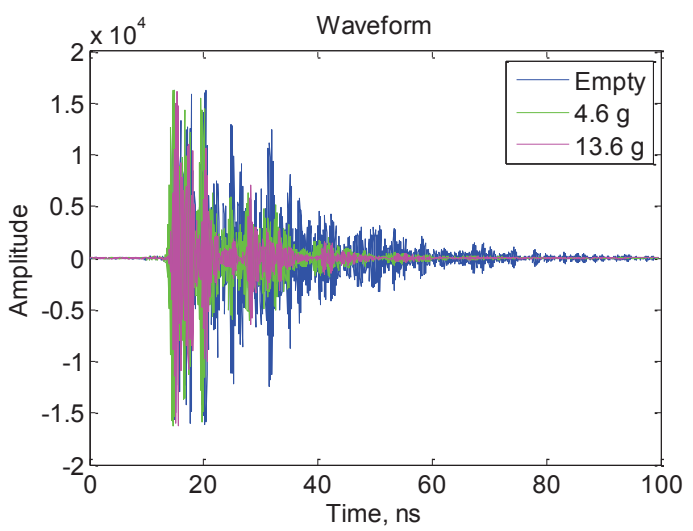

Fig. 4. UWB waveforms for three DPFs loaded with different amounts of soot.

The UWB pulse waveforms recorded during the communication between two commercial PulsON P410 modules via the interior of the DPF-loaded metal housing look substantially different for the various DPFs (Fig. 4). In particular, multiple-transit signals inside the catalyst housing (occurring after $18 \mathrm{~ns}$ in Fig. 4) are the more attenuated, the higher the soot load is. This is explained by the fact that the conducting soot introduces losses into the system. Thus, a wave packet is attenuated each time it traverses the soot-loaded DPF, and this attenuates multiple-transit signals more than the single-transit (direct input-to-output) signal. In Fig. 4 the vertical scale represents A-D (analog-to-digital) counts proportional to volts as output by the analog-to-digital converter (ADC) in the UWB chip. 


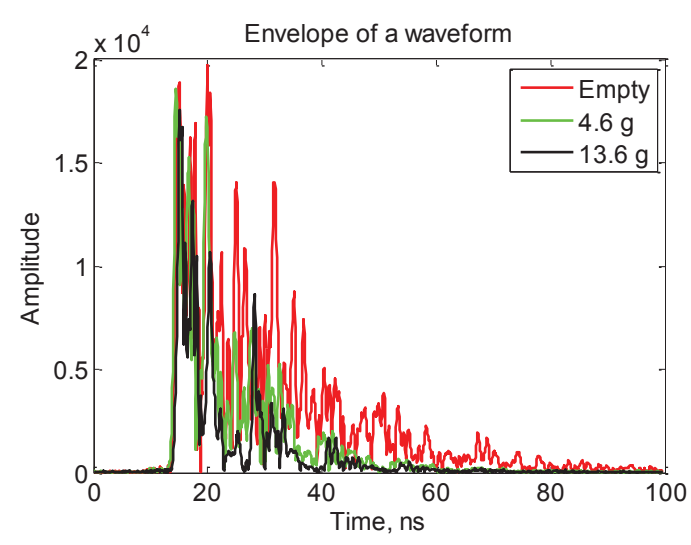

Fig. 5. Envelopes of the time-domain responses from Fig. 4.

The envelopes $e(t)$ of the time-domain responses shown in Fig. 4 have been computed as the magnitude of the analytic signal constructed from the original time response $u(t)$ by adding to it its Hilbert transform [8]:

$e(t)=|u(t)+j \hat{u}(t)| w$ ith $\hat{u}(t)=\frac{1}{\pi} \int_{-\infty}^{\infty} \frac{u(\tau)}{t-\tau} \mathrm{d} \tau$.

(Of course, all signals are discrete-time.)

The result is shown in Fig. 5. One clearly observes a substantial difference in the signal envelopes depending on the soot content in the DPF. In particular, this can be seen starting from the second peek at around $16 \mathrm{~ns}$. The soot loading state is not observable via the first peak because of the technical characteristics of the equipment (at the beginning of the received UWB pulse, the Tx pulse coupling through the Tx switch is showing through).

To quantify the differences, we calculated the convolution of the envelope functions for different DPF states, as always defined by

$c_{i j}(t)=\int_{-\infty}^{+\infty} e_{i}(\tau) e_{j}(t-\tau) \mathrm{d} \tau$

(Fig. 6). The effect of the soot load on the curves is clearly seen. Multiple-transit signals are more attenuated with increasing soot load. The amplitude of the convolved signals at $50 \mathrm{~ns}$ is around $14 \times 10^{9}$ (A-D counts) for the empty/4.6-g case, but only $10 \times 10^{9}$ for the empty/13.6-g case.

The difference is observable by the width of the convolution curves as well. Measured at an amplitude of $4 \times 10^{9}$, this width amounts to $48 \mathrm{~ns}$, $40 \mathrm{~ns}$, and $23 \mathrm{~ns}$, respectively, for the three cases.

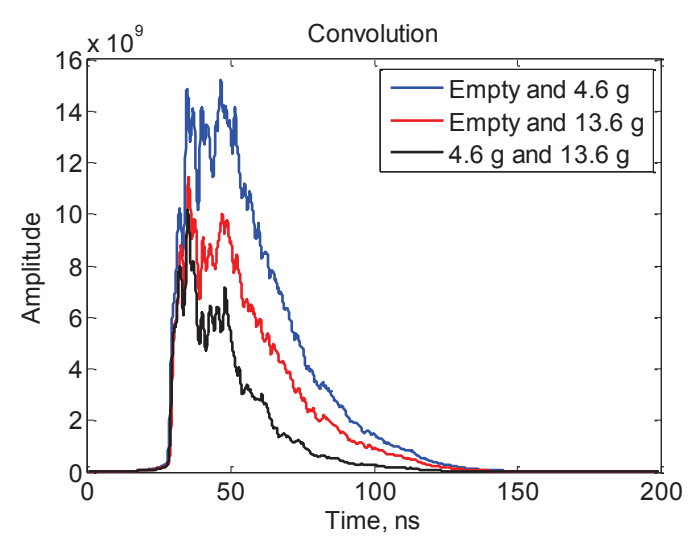

Fig. 6. Convolution of the envelopes of the timedomain responses (Fig. 5).

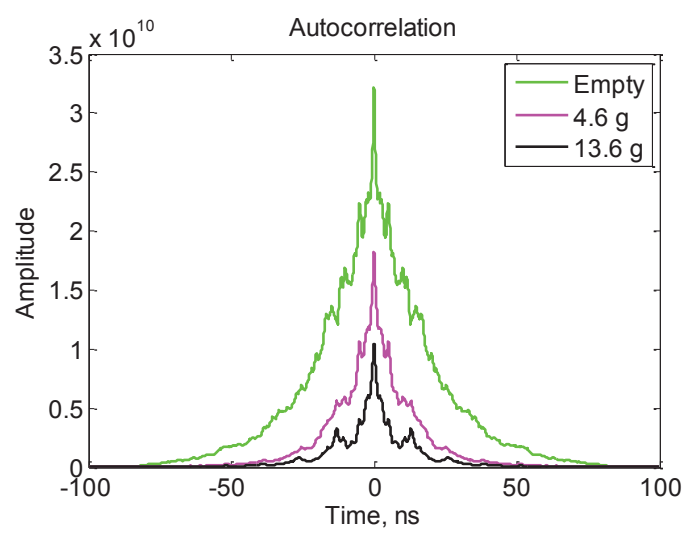

Fig. 7. Autocorrelation functions of the envelopes shown in Fig. 5.

We also calculated the cross-correlation of the envelope functions, given by

$\Phi_{i j}(\tau)=\int_{-\infty}^{+\infty} e_{i}(t) e_{j}(t+\tau) \mathrm{d} t$

For $i=j$, this turns into the auto-correlation $\Phi_{i i}(\tau)$ of the envelope function (Fig. 7). The maximum amplitude is $3.2 \times 10^{10}, 1.8 \times 10^{10}$, and $1 \times 10^{10}$ for the empty DPF, the DPF loaded with $4.6 \mathrm{~g}$ soot, and the DPF loaded with $13.6 \mathrm{~g}$, respectively. Again, the differences are observable via the signal width, too.

Finally, Fig. 8 shows the cross-correlation functions. The amplitudes and mean values of the peaks are among the parameters suitable for a DPF state estimation algorithm. The maximum amplitude was $2.2 \times 10^{10}, 1.5 \times 10^{10}$, and $1.3 \times 10^{10}$ for the cross-correlations of the three envelope signals from Fig. 5 . The mean values were $2.8 \times 10^{9}, 1.8 \times 10^{9}$, and $1.1 \times 10^{9}$ for the three cases, respectively.

Owing to the rapid amplitude variation of the envelope curves in Fig. 5, it makes sense to first smooth them before further processing (Fig. 9). 


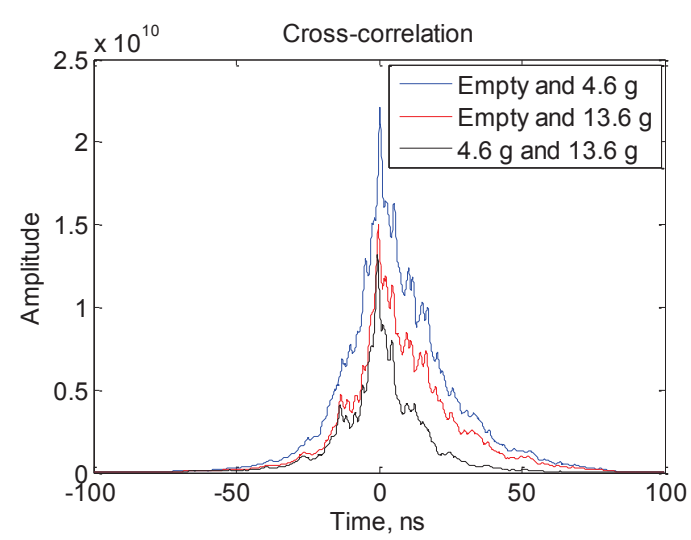

Fig. 8. Cross-correlation functions of the envelopes shown in Fig. 5.

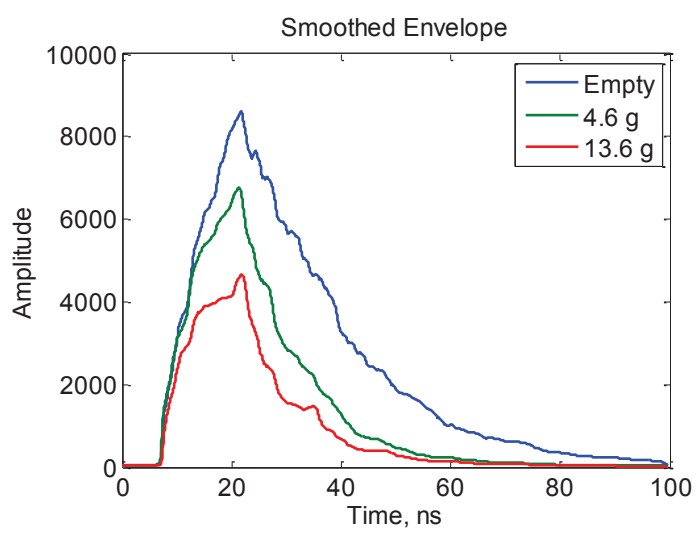

Fig. 9. Smoothed envelopes of the time-domain responses from Fig. 4.

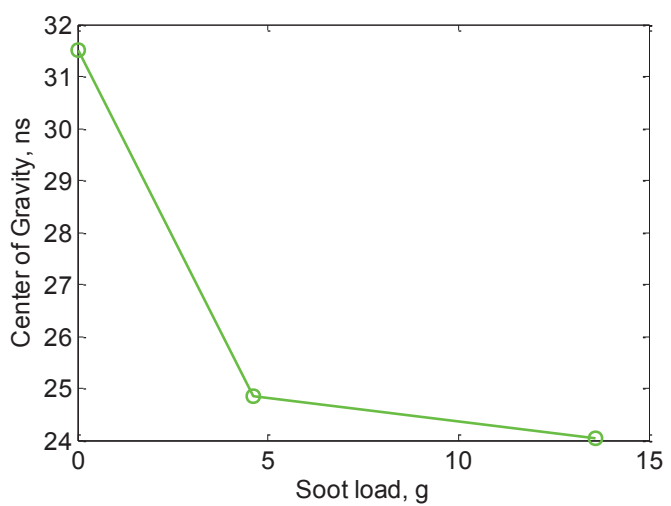

Fig. 10. Time coordinate of the center of gravity of the area bounded by the smoothed envelopes and the time axis.

One possible way of processing is to correlate certain features of these smoothed curves with the DPF soot load. This is shown in Fig. 10 for the center of gravity of the areas bounded by the curves in Fig. 9 and the time axis. It is obvious that this characteristic curve may be used to estimate the soot load from the measured time-domain response of the UWB system. It is also obvious that the sensitivity of this measurement system decreases with increasing soot load.

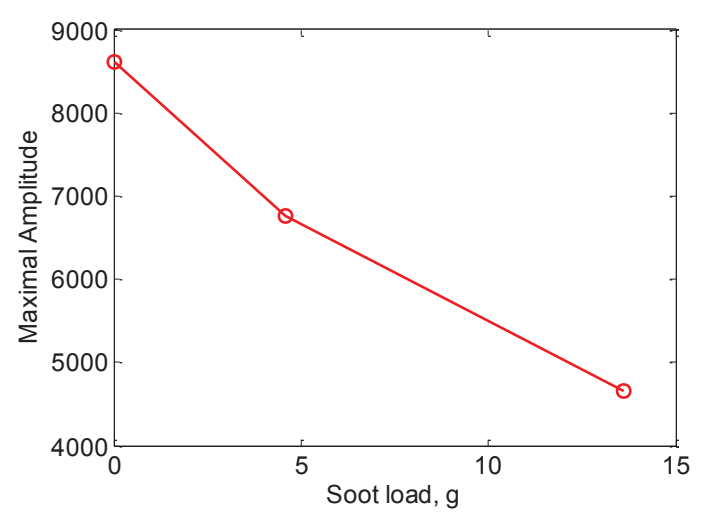

Fig. 11. Maximum value of the smoothed envelopes.

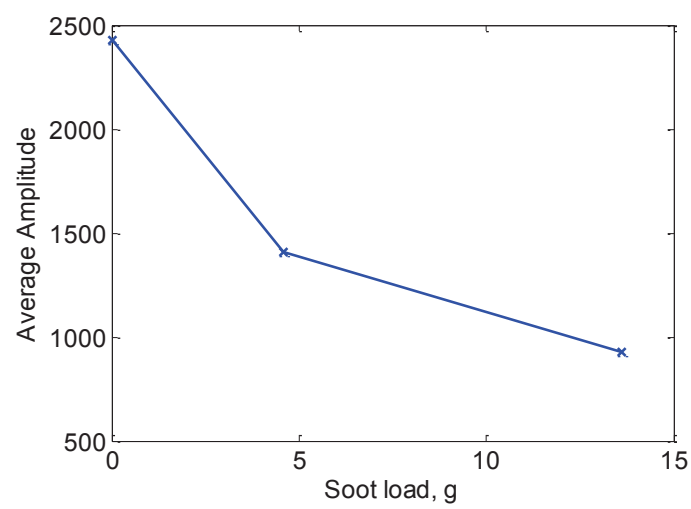

Fig. 12. Average value of the smoothed envelopes.

Along the same line of reasoning, we have computed the characteristic curves linking the maximum and the average values of the smoothed envelopes with the DPF soot load (Figs. 11, 12).

While the overall behavior of the characteristic curves in Figs. 10 through 12 is quite similar, there are differences in detail. It appears as if small soot loads can be better resolved (measured with a higher sensitivity) by way of the center-of-gravity feature (Fig. 10) than by the other features. This would be quite interesting, but, in our opinion, it is too early to draw such conclusions as the number of measurements we have performed so far is much too small to allow any substantial insight into the statistics of the measurement approach.

Besides, it is also important to note that the curves will look different with different transmitting power because of receiver sensitivity. This, too, will have to be investigated. 


\section{Conclusions}

We have reported on a novel approach for the monitoring of exhaust gas aftertreatment systems, such as DPFs, SCRs, and TWCs, the most attractive feature of which is that it is based on a cost-effective modular architecture. Hence, it can be implemented in situ, e.g., in a car.

In the approach proposed, the interior of the catalyst metal housing serves as a communication channel between two wireless communication nodes. To be more specific, we investigated a UWB-based architecture. It was demonstrated experimentally by way of various soot-loaded DPFs, that the catalyst chemistry (i. e., the soot load of the DPF) affects the channel characteristics strongly enough to allow the identification of the DPF state from the signal waveform details.

In particular, we were able to show that, in principle, certain features of the received timedomain signals depend in a manner on the soot load which allows the inversion of the feature vs. soot load characteristics. Among these features were the position of the center of gravity of the area bounded by the signal envelope and the time axis, the maximum value, and the average value of the envelopes.

\section{Acknowledgement}

The authors thankfully acknowledge financial support by the German Research Foundation (DFG) under grants Fi 956/3-2 and Fi 956/5-1.

\section{References}

[1] M. Feulner et al., Topics in Catalysis 56, Issues 1-8, 483-488 (2013); doi: 10.1007/s11244-0130002-9

[2] D. Rose, T. Boger, SAE Technical Paper 200901-1262 (2009); doi: 10.4271/2009-01-1262

[3] S. Schödel, R. Moos, M. Votsmeier, G. Fischerauer, IEEE Trans. CST, Vol. 22, No. 6, 23462353 (Nov. 2014); doi: 10.1109/tcst.2014. 2305576

[4] M. V. Twigg, Applied Catalysis B: Environmental 70, 2-15 (2007); doi: 10.1016/j.apcatb.2006.02. 029

[5] I. Motroniuk, R. Królak, R. Stöber, G. Fischerauer, 5th IMEKO TC19 Symp. Environmental Instrum. Meas., Chemnitz, 122-126 (Sept. 2324, 2014)

[6] G. Fischerauer et al., Frequenz 62, 180-184 (2008); doi: 10.1515/freq.2008.62.7-8.180

[7] G. Fischerauer, M. Förster, R. Moos, Meas. Sci. Technol., vol. 21, issue 3, 035108 (March 2010); doi: 10.1088/0957-0233/21/3/035108

[8] H. Marko, Methoden der Systemtheorie (in German). Berlin etc.: Springer, 114 ff. (21982). 